\title{
TRANS-ENDOTHELIAL INSULIN TRANSPORT IS IMPAIRED IN SKELETAL MUSCLE CAPILLARIES OF OBESE MALE MICE.
}

\author{
Ian M Williams ${ }^{1}$, P Mason McClatchey ${ }^{1}$, Deanna P Bracy ${ }^{1,2}$, Jeffrey S Bonner ${ }^{3}$, Francisco A \\ Valenzuela $^{3}$, David H Wasserman ${ }^{1,2}$ \\ ${ }^{1}$ Department of Molecular Physiology and Biophysics, Vanderbilt University, Nashville, Tennessee, \\ USA \\ ${ }^{2}$ Mouse Metabolic Phenotyping Center, Vanderbilt University, Nashville, Tennessee, USA \\ ${ }^{3}$ Lilly Research Laboratories, Indianapolis, Indiana, USA
}

\section{Abstract}

Objective: The continuous endothelium of skeletal muscle capillaries regulates insulin's access to skeletal myocytes. Whether impaired trans-endothelial insulin transport (EIT) contributes to skeletal muscle (SkM) insulin resistance (IR), however, is unknown.

Methods: Male and female C57/B16 mice were fed either chow or high fat diet (HFD) for 16 weeks. We used intravital microscopy to measure EIT in SkM capillaries, electron microscopy to assess endothelial ultrastructure, and glucose tracers to measure indices of glucose metabolism.

Results: We found that DIO male mice have a $\sim 15 \%$ reduction in EIT compared to lean mice. Impaired EIT was associated with a $45 \%$ reduction in endothelial vesicles. Despite impaired EIT, hyperinsulinemia sustained delivery of insulin to the interstitial space in DIO male mice. Even with sustained interstitial insulin delivery, DIO male mice still showed SkM IR indicating severe myocellular IR in this model. Interestingly, there was no difference in EIT, endothelial ultrastructure or SkM insulin sensitivity between lean and HFD-fed female mice.

Conclusions: These results suggest that, in male mice, obesity results in ultrastructural alterations to the capillary endothelium which delay EIT. Nonetheless, the myocyte appears to exceed the endothelium as a contributor to SkM IR in DIO male mice.

\section{Keywords}

Skeletal muscle; Insulin resistance; Endothelial transport; Endothelial function; Obesity

\footnotetext{
Corresponding Author: Ian M Williams, PhD, Department of Molecular Physiology and Biophysics, Vanderbilt University, 823 Light Hall, 2215 Garland Avenue, Nashville, TN 37232-6602, Phone 615-343-7336, ianmw@ @tanford.edu.

Author contributions: The project was conceived and designed by IMW and DHW. IMW, JSB, DPB, and FAV performed experiments and collected data. IMW wrote the manuscript. Manuscript edits and discussions were contributed by PMM, JSB, FAV, and DHW. IMW is the guarantor of this work and, as such, had access to all data and takes full responsibility for the work as a whole. Disclosure: While this study was being conducted, F.A.V. and J.S.B were employees of Eli Lilly and Company, a pharmaceutical company. Eli Lilly generously provided the fluorescent insulin probe (INS-647) used in the study. The authors declare no other competing financial interests.
} 


\section{INTRODUCTION}

Skeletal muscle (SkM) comprises the vast majority of insulin-sensitive tissue. As such, it is a major site of insulin resistance (IR) during obesity $(1,2)$. Furthermore, SkM IR precedes the development of type 2 diabetes (3). Determining the mechanism of SkM IR, therefore, is critical to developing therapies that restore metabolic function in individuals with obesity.

Insulin action in SkM depends on insulin delivery to the myocyte, binding of insulin to the insulin receptor, and activation of signaling cascades. Following its appearance in the systemic circulation, insulin stimulates endothelium-dependent relaxation of SkM arteries and arterioles (4). This vasodilation enhances SkM perfusion and is thought to increase the capillary surface area available for glucose and insulin exchange (5). Subsequently, insulin must transit the continuous endothelium of SkM capillaries by a non-saturable, fluid-phase transport process $(6,7)$. The endothelium is a significant barrier which restricts insulin's access to SkM and, thus, is a key regulator of SkM insulin action $(8,9)$.

Impaired SkM insulin signaling (reviewed in Ref 12) and reduced glucose transport $(11,12)$ are features of obesity, IR, and type 2 diabetes. These characteristics have been attributed to the toxic lipid accumulation (13), mitochondrial dysfunction (14), and inflammation (15) that occurs in individuals with obesity. It has also been hypothesized that this decreased insulin signaling is caused by reduced microvascular delivery of insulin to myocytes (9). That is, pathological processes occurring outside the myocyte may contribute to SkM IR.

Several investigators have utilized techniques such as microdialysis and lymph sampling to measure SkM insulin delivery $(16,17)$. Many $(18-20)$, but not all $(21,22)$, of these studies have found that SkM insulin delivery is reduced in humans and animal models with obesity and type 2 diabetes. This observation suggests that microvascular dysfunction may contribute to SkM IR. The delivery of insulin to SkM is determined by 1) the surface area for insulin exchange and 2) the rate that insulin transits the capillary endothelium. Several studies have demonstrated that insulin-stimulated SkM perfusion is reduced in humans with obesity, IR, and type 2 diabetes (as reviewed in Ref 5). This decrease in perfusion may reduce the surface area for insulin exchange and, consequently, microvascular insulin delivery. Whether the rate of trans-endothelial insulin transport (EIT) is altered during obesity and IR, however, is unknown.

We hypothesized that EIT is reduced in the setting of obesity and SkM IR. To address this hypothesis, we utilized the high-fat diet (HFD)-fed mouse model of obesity and IR (23). We found that HFD-fed male mice had a $45 \%$ reduction in the density of capillary endothelial vesicles, the putative transport vehicles for EIT. This alteration to capillary endothelial ultrastructure was associated with a 15\% reduction in EIT, as determined by quantitative intravital microscopy (6). Conversely, female mice were protected from the effects of HFD on adiposity, endothelial ultrastructure, EIT, and SkM insulin sensitivity.

\section{METHODS}

Animal procedures were approved by the Vanderbilt Institutional Animal Care and Use Committee and conducted in accordance with the NIH Guide for the Care and Use of 
Laboratory Animals. Methodological details can be found below and in the Online Supporting Information.

\section{Mouse models}

Male and female 6-week old C57B1/6 mice were randomized to either standard chow (5001 Laboratory Rodent Diet; Lab Diet) or HFD (60\% calories from fat; Bioserv F3282) for 16 weeks.

\section{Electron microscopy}

Tissues were fixed by perfusing anesthetized mice with $2.5 \%$ glutaraldehyde in $0.1 \mathrm{~mol} / \mathrm{l}$ cacodylate buffer through a catheter inserted into the left ventricle. Sections were imaged using a Philips/FEI Tecnai T12 electron microscope (ThermoFisher Scientific) and quantified using ImageJ (Online Supporting Information).

\section{Intravital microscopy}

Intravital imaging of fluorescent probes (insulin-647 - INS-647; albumin-647 - Alb-647; 2 megadalton tetramethylrhodamine dextran - rho-dex) was performed as described previously (6). Of note, INS-647 behaves identically to native insulin in terms of receptor-binding capacity, in vivo and ex vivo bioactivity, and pharmacokinetics (6). Fluorescent probes were infused through an indwelling jugular vein catheter and imaged in the lateral gastrocnemius using an LSM780 confocal microscope (Zeiss). INS-647 and Alb-647 concentrations were measured in the capillary, interstitial (i.e. peri-capillary), and extravascular spaces as a function of time using an automated Otsu-based thresholding algorithm (6). The interstitial space was defined as the region emanating radially $1-3 \mu \mathrm{m}$ from the capillary wall. We measured extravascular INS-647 by dilating the vascular mask by $1 \mu \mathrm{m}$ and inverting it to segment the entire extravascular space in the field of view.

\section{Insulin tolerance tests}

To test whether SkM IR could be detected in DIO male mice, we performed intravenous insulin tolerance tests using the same protocol as intravital microscopy (i.e. anesthetized, $4 \mathrm{U} / \mathrm{kg}$ insulin tolerance tests). Tissue glucose clearance was calculated from plasma $2\left[{ }^{14} \mathrm{C}\right]$ deoxyglucose $\left(2\left[{ }^{14} \mathrm{C}\right] \mathrm{DG}\right)$ and tissue $2\left[{ }^{14} \mathrm{C}\right] \mathrm{DG}-6$-phosphate $\left(2\left[{ }^{14} \mathrm{C}\right] \mathrm{DGP}\right)$ measurements as described previously (24).

\section{Single-cell RNA sequencing}

Measurements of insulin receptor mRNA levels in single endothelial cells (ECs) from various mouse tissues were obtained using the publicly available Tabula Muris dataset (25). These measurements were obtained from 4 male and 3 female 10-15 week old C57B1/6JN mice.

\section{Statistics}

Statistical analysis was performed using Prism 7 (Graphpad). Data are presented as mean \pm standard error of the mean. Groups were compared using either unpaired Student's t-tests or two-way repeated measures ANOVA. 


\section{RESULTS}

\section{HFD causes ultrastructural alterations to the SkM capillary endothelium of male mice}

Obesity and SkM IR are associated with endothelial dysfunction (26). To determine whether this may affect EIT, we characterized capillary endothelial ultrastructure in a mouse model of diet-induced obesity (DIO) and SkM IR. Lean and DIO mouse models were generated by feeding male mice chow or HFD for 16 weeks. As expected, DIO mice weighed 52\% more than their lean counterparts (Figure S1A) and had a 2.5 -fold increase in body fat percentage (Figure S1B).

Using transmission electron microscopy, we observed that the density of vesicles in the endothelium was reduced by $\sim 45 \%$ in DIO male mice (Figure 1A-B). These mice showed a profound increase in the proportion of capillaries with very few endothelial vesicles, and a decrease in the number of capillaries with a higher density of vesicles (Figure 1C). Neither the diameter of vesicles (Figure 1D), nor their circularity (Figure 1E), however, were altered in DIO male mice. The localization of vesicles within the endothelium was unchanged between groups (Figure 1F), although we were underpowered to detect subtle differences (Figure S2). We also did not observe any differences in basement membrane thickness (Figure 1G) or endothelial volume (Figure 1H) between lean and DIO male mice. This observation of reduced endothelial vesicles, which can be either distinct vesicular transporters or form trans-endothelial channels, raises the possibility that EIT may be impaired in DIO male mice.

\section{HFD impairs trans-endothelial insulin transport in male mice}

Given that DIO male mice have fewer endothelial vesicles, the putative transport vehicle for EIT, we hypothesized that EIT would also be reduced in DIO male mice. Using intravital insulin imaging, we observed that EIT is impaired in DIO male mice (Figure 2). Namely, the rate at which plasma INS-647 equilibrated with the interstitial space was $\sim 15 \%$ slower in DIO mice compared to lean mice (Figure 2D\&E). Following the INS-647 bolus, capillary INS-647 levels were consistently higher in DIO male mice (Figure 2B\&C, Table 1). The clearance of plasma INS-647, however, was not significantly different between groups (Figure S3). Furthermore, there was no significant relationship between capillary plasma INS-647 levels and trans-endothelial insulin equilibration (Figure S4), suggesting that the reduced EIT in DIO male mice was not caused by elevated plasma INS-647. Consistent with higher levels of plasma INS-647, DIO male mice also had higher absolute interstitial INS-647 (Figure 2B\&C, Table 1). However, the magnitude of the increased interstitial INS-647 in DIO male mice was lower than that of the increase in plasma INS-647 (Figure 2C). These findings indicate that, while DIO male mice had higher plasma INS-647, a lower fraction of plasma INS-647 was able to access the interstitial space.

EIT is one of three variables that control SkM insulin delivery. The other two variables are plasma insulin concentration and the capillary surface area for insulin exchange. We found that neither plasma-perfused surface area (Figure S5A\&B) nor capillary diameter (Figure S5C\&D) were affected by HFD. This suggests that the surface area for insulin exchange is the same in lean and DIO males. To determine whether the reduced EIT in DIO males 
affects total insulin delivery, we measured INS-647 in the extravascular space. We found that extravascular INS-647 levels were consistently higher in DIO male mice (Figure 2B\&C, Table 1). These findings indicate that, in DIO male mice, SkM insulin delivery is sustained by hyperinsulinemia in the face of impaired EIT.

Following intravital microscopy experiments, we observed that DIO male mice had higher blood glucose levels (Figure S6A) and lower accumulation of $2\left[{ }^{14} \mathrm{C}\right]$ deoxyglucose-6phosphate $\left(2\left[{ }^{14} \mathrm{C}\right] \mathrm{DGP}\right)$ in the gastrocnemius (Figure S6B). These findings suggest that DIO male mice have whole-body and SkM IR. However, the rate of glucose entry into SkM cannot be assessed from $2\left[{ }^{14} \mathrm{C}\right] \mathrm{DGP}$ accumulation alone. To connect impaired EIT to SkM IR, we performed insulin tolerance tests with $2\left[{ }^{14} \mathrm{C}\right]$ deoxyglucose $\left(2\left[{ }^{14} \mathrm{C}\right] \mathrm{DG}\right)$ in a separate cohort of DIO male mice treated in the same manner as mice that underwent intravital microscopy (Figure 3). As expected, DIO male mice had an impaired glucose-lowering response to insulin (Figure 3A\&B), despite similar levels of plasma insulin (Figure 3C\&D). Furthermore, $2\left[{ }^{14} \mathrm{C}\right] \mathrm{DG}$ clearance by the soleus, gastrocnemius, and vastus muscles was reduced in DIO male mice (Figure 3E), indicating impaired glucose entry into muscle.

\section{Venules, but not capillaries, accumulate insulin and dextran}

Our previous work demonstrated that insulin transits the capillary endothelium by a fluidphase transport mechanism that does not involve the insulin receptor (6). To corroborate this finding, we assessed the expression of insulin receptor mRNA in endothelial cells (ECs) from various mouse organs using the Tabula Muris single-cell RNA sequencing dataset (25). We found that insulin receptor expression was undetectable in the majority (84\%) of SkM ECs (Figure 4A). Conversely, the insulin receptor is more highly expressed in brain and liver ECs (Figure 4A). The lack of detection of insulin receptor expression in SkM ECs corroborates our previous finding that insulin does not cross SkM capillary endothelium by insulin receptor-mediated transport (6).

Insulin receptor-independent transport may involve either non-specific vesicular pinocytosis or convective movement through trans-endothelial channels. If insulin were being trafficked across the endothelium by discrete vesicular transporters, we would expect to observe INS-647 accumulation in the lining of the endothelium. We did not detect accumulation of either INS-647 or rho-dex in the capillary endothelium in the present study (Figure 2A, $4 \mathrm{~B} \& \mathrm{C}$, and $7 \mathrm{~A})$ nor in our previous work $(6,27)$. However, we did observe a striking accumulation of INS-647 and rho-dex in cells lining post-capillary venules in both lean and DIO male mice (Figure 4B\&C). Thus, we can detect vascular INS-647 accumulation by intravital microscopy should it exist. These data support the possibility that, in capillaries, insulin is not transported by distinct vesicular transporters but rather moves through transendothelial channels. We posit that the decrease in endothelial vesicles observed in DIO male mice (Figure 1B) indicates a reduction in trans-endothelial channels available for EIT.

\section{No difference in trans-endothelial albumin equilibration between lean and DIO male mice}

To determine whether the altered endothelial ultrastructure observed in DIO male mice restricts the movement of other plasma-borne molecules, we measured the trans-endothelial equilibration of albumin-647 (Alb-647; Figure 5). Albumin (hydrodynamic radius $\sim 3.5 \mathrm{~nm}$ ) 
is a significantly larger protein than insulin (hydrodynamic radius $\sim 1.3 \mathrm{~nm}$ ) and does not leak from capillaries unless endothelial barrier function is compromised (28). In contrast to INS-647, the levels of Alb-647 were stable in the plasma and interstitial space over the course of the 15-minute experiment (Figure 5B\&C). The levels of plasma and interstitial Alb-647 were slightly elevated, although not significantly different, in DIO compared to lean male mice (Table 1). We did not observe any differences in the ratio of plasma to interstitial Alb-647 between lean and DIO male mice (Figure 5D\&E), indicating that capillary permeability to Alb-647 is unchanged by diet.

\section{HFD does not alter SkM capillary endothelial ultrastructure nor trans-endothelial insulin transport in female mice}

We next tested whether the effects of HFD on endothelial ultrastructure and EIT were similar in female mice. Female mice fed a HFD for 16 weeks weighed $30 \%$ more than their chow-fed counterparts (Figure S7A), but did not have a significantly higher percentage of body fat (Figure S7B).

In contrast to male mice, we did not observe any difference in endothelial vesicular density between chow and HFD-fed female mice (Figure 6A\&B). The distribution of capillary vesicular densities appeared to shift from a normal distribution in chow-fed females to a more bimodal distribution in HFD-fed females (Figure 6C). This subtle shift in capillary vesicular distribution may represent an early stage of HFD-induced endothelial dysfunction. In general, however, female mice are largely protected from the effects of HFD on capillary endothelial ultrastructure.

Given that endothelial ultrastructure is unaffected by HFD in female mice, we hypothesized that EIT would also be unchanged in these mice. Indeed, we did not observe any difference in EIT between chow and HFD-fed females (Figure 7). Specifically, there was no change in the dissipation of the plasma/interstitial INS-647 gradient (Figure 7D\&E). Plasma and interstitial INS-647 levels were slightly, but not significantly, higher in HFD-fed females following the INS-647 bolus (Figure 7B\&C, Table 1). No differences in either average plasma-perfused surface area (Figure S8A\&B) or capillary diameter (Figure S8B\&C) were observed. As expected from the lack of differences in capillary surface area (Figure S8B) and EIT (Figure 7D\&E), there was no change in total extravascular INS-647 delivery (Figure 7B\&C, Table 1) in HFD-fed females. Following intravital microscopy, we did not observe any difference in terminal blood glucose (Figure S9A). HFD-fed females had a nonsignifcant reduction in $2\left[{ }^{14} \mathrm{C}\right] \mathrm{DGP}$ accumulation in the gastrocnemius (Figure S9B). These findings suggest that, in contrast to males, 16 weeks of HFD neither impedes EIT nor causes SkM IR in female mice.

\section{DISCUSSION}

We found that 16 weeks of HFD causes a profound reduction in the number of endothelial vesicles in SkM capillaries of male mice. These ultrastructural alterations to the endothelium are associated with impaired EIT in vivo. Of note, this is the first study to directly observe impaired EIT in a mouse model of obesity and SkM IR in vivo. Interestingly, however, interstitial insulin delivery was maintained in DIO male mice by elevated plasma insulin. 
The finding that DIO male mice still show severe SkM IR even with maintained interstitial insulin indicates that myocytes are the major contributor to SkM IR in this model. On the other hand, female mice were protected from the effects of HFD on endothelial ultrastructure, EIT, and SkM insulin sensitivity. In summary, these findings indicate that while HFD impairs EIT in male mice, interstitial insulin delivery can be sustained through hyperinsulinemia, a characteristic of metabolic disease.

Most previous studies, but not all (21), have demonstrated that SkM insulin delivery is reduced in humans (18) and animal models $(19,20)$ with obesity. SkM insulin delivery depends on the capillary surface area for insulin exchange and the rate of insulin movement across the endothelium. Several studies have shown that insulin-stimulated SkM perfusion is reduced in obesity (as reviewed in Ref 5). The contribution of EIT to the reduced insulin delivery observed in obesity, however, has been unclear due to an inability to make direct measurements in vivo. We recently developed an intravital microscopy technique to directly visualize and quantify EIT in individual SkM capillaries in vivo (6). This method has major advantages over in vitro studies of cultured ECs. Namely, cultured ECs are very different than capillaries in vivo with respect to microenvironment, protein expression, permeability, and the mechanism of insulin transport $(6,29-31)$. Utilizing intravital microscopy, we demonstrate that EIT is impaired in SkM capillaries of DIO male mice.

We observed a $15 \%$ reduction in EIT in DIO male mice. The implication of this reduction in EIT would presumably be a reduction in total delivery of insulin to SkM. Instead, we found that interstitial INS-647 levels were actually elevated in DIO male mice. This finding highlights that, in addition to surface area for insulin exchange and EIT, capillary plasma insulin is an important determinant of SkM insulin delivery. In the current study, higher capillary plasma INS-647 in DIO male mice compensate for reduced EIT to maintain interstitial and extravascular INS-647 delivery. These observations parallel those of Bergman and colleagues who found that, during hyperinsulinemic-euglycemic clamps in HFD-fed dogs, higher arterial insulin levels overcame impaired insulin delivery to maintain insulin levels in the lymph (20). Furthermore, our finding that the kinetics of, but not capacity for, SkM insulin delivery is impaired in DIO male mice corroborates similar findings in humans $(16,18,22)$.

Capillary plasma INS-647 was likely higher in DIO mice because the dose of INS-647 was normalized to total body mass and plasma volume does not scale with body mass in obese mice (32). That is to say, more units of INS-647 were administered per unit volume in DIO mice, resulting in elevated capillary plasma INS-647. Increased capillary plasma INS-647 alone does not affect EIT because 1) insulin efflux is not saturable (6), and 2) there is no relationship between capillary INS-647 and EIT. In contrast to the measurements of capillary INS-647 with intravital microscopy, we did not observe any differences in arterial insulin between lean and DIO male mice during insulin tolerance tests. This suggests that, in DIO male mice, more insulin was diverted from the systemic arterial circulation to the gastrocnemius microvasculature. One feature of our study that should be noted is that, due to the sensitivity limits of fluorescence microscopy, we use a high dose of INS-647 which produces supraphysiological plasma insulin levels. However, EIT is non-saturable and is 
independent of the dose of INS-647 used (6). Thus, our findings reflect an alteration in barrier function that is inherent to the capillary endothelium of DIO male mice.

Despite higher levels of extravascular insulin in DIO male mice, they still displayed SkM IR. This reflects cellular IR at the level of the myocyte. Thus, myocellular IR is compounded by reduced EIT to more severely impair insulin action in SkM during obesity. Taken together, our data demonstrate that myocellular IR is a more substantial contributor to SkM IR than deficits in EIT in HFD-fed male mice.

The delayed EIT in DIO male mice is associated with a profound reduction in endothelial vesicular density. We suspect that the vast majority of these vesicles are caveolae as their diameter $(\sim 60-65 \mu \mathrm{m})$ is identical to the nominal diameter of caveolae and the majority of endothelial vesicles are caveolae (33). Our observation of reduced endothelial vesicles is consistent with the demonstration that the number of endothelial vesicles in coronary arterioles is reduced in humans with established type 2 diabetes (34). Furthermore, Barrett and colleagues have shown that the expression and phosphorylation of caveolin-1, the main protein component of caveolae, are required for the uptake of insulin by cultured aortic endothelial cells (35-37). Thus, endothelial caveolae appear to be involved in TIE and reduced in states of metabolic disease. Finally, while we did not observe any difference in basement membrane width between groups, we cannot rule out the possibility that altered chemical or steric interactions between insulin and basement membrane proteins $(38,39)$ may also hinder EIT in DIO male mice.

Endothelial vesicles can serve as macromolecular transporters (33) which may carry insulin (40). It is unclear whether these vesicles are distinct, transcytotic carriers or if they represent pores in the endothelium (41). We hypothesize that these capillary endothelial vesicles are likely components of pore networks for two reasons. First, we previously showed that EIT is not receptor-mediated nor saturable in vivo (6). We validated these findings in this study by showing that insulin receptor expression cannot be detected in the majority of SkM ECs. While the Smart-Seq2 protocol used by the Tabula Muris investigators has a lower detection limit of $<50$ mRNA molecules per cell (42), we cannot rule out the possibility that SkM ECs express the insulin receptor at very low levels. Nonetheless, the transport of insulin through endothelial pores is more compatible with the non-saturable nature of insulin transport. The presence of a non-saturable yet active, energy-requiring transcytotic transport mechanism seems unlikely. Furthermore, if individual vesicles were actively taking up macromolecules and being transcytosed across the endothelium, we may expect to see molecular accumulation in the capillary endothelium. Unlike studies utilizing cultured ECs (43), we do not observe the accumulation of either INS-647 or rho-dex in the lining of SkM capillaries. It is possible that vesicular transcytosis occurs so rapidly in capillaries as to preclude endothelial accumulation. We did, however, observe accumulation of these molecules in the cells lining venules, indicating that vesicular transcytosis may be a relevant transport mechanism in larger blood vessels. In summary, we hypothesize that, in DIO male mice, the number of capillary endothelial pores available for insulin exchange is reduced, thereby impairing EIT. 
Intravital microscopy also allows for the measurement of indices of microvascular structure and hemodynamics. We did not observe any difference in plasma-perfused surface area or capillary diameter between lean and DIO male mice. Furthermore, the administration of insulin did not affect plasma-perfused surface area in either group. That is to say, we did not observe any capillaries which had no plasma at rest that were then subsequently perfused by plasma after insulin administration. As insulin is transported in plasma, these findings suggest that there is no effect of HFD or insulin on the capillary surface area available for insulin exchange. It should be noted that these measurements of plasma volume are much different than the index of red blood cell (RBC) volume and velocity afforded by contrast-

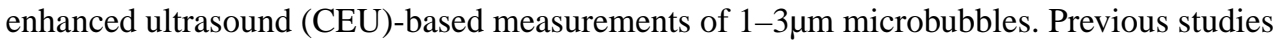
using this technique concluded that 1) insulin increases RBC volume and velocity in SkM and 2) this effect of insulin is blunted in states of $\operatorname{SkM~IR}(5,19)$. Given that plasma and RBCs have different distribution patterns in the microcirculation (44), it is possible that the effects of insulin and HFD on RBC and plasma distribution are also divergent. Namely, while insulin may increase and obesity may decrease microbubble distribution volume and velocity (5), neither insulin nor obesity alter SkM plasma-perfused surface area.

In contrast to male mice, female mice were protected from the effects of HFD on EIT and endothelial ultrastructure. The most likely explanation for this finding is that, while HFD-fed female mice were heavier than chow-fed mice, they did not have a significantly higher percentage of body fat. We surmise that female mice did not accumulate as much adipose tissue as male mice because they consumed less of the HFD, as has been demonstrated previously (45). These investigators also found that, similar to the present study, 15 weeks of HFD causes weight gain but only a small increase in body fat percentage in female mice (45). Consistent with unaltered EIT, neither SkM insulin sensitivity nor endothelial vesicular density were altered by HFD in female mice.

In summary, we show for the first time that insulin movement across the SkM capillary endothelium is impaired in a mouse model of DIO and IR. This impairment in EIT is associated with profound alterations to endothelial ultrastructure. These findings demonstrate that the capillary endothelium limits the delivery of insulin to insulin resistant skeletal myocytes. Potential strategies to accelerate EIT include maintaining endothelial ultrastructure, widening inter-endothelial junctions, or increasing capillary surface area for exchange. These approaches may be useful in enhancing the delivery of insulin to SkM for the treatment of IR or in generating fast-acting insulin analogs for diabetics.

\section{Supplementary Material}

Refer to Web version on PubMed Central for supplementary material.

\section{Acknowledgments.}

The authors thank Freyja James (Department of Molecular Physiology and Biophysics, Vanderbilt University) for performing catheterization surgeries. The authors gratefully acknowledge the use of services provided by the Vanderbilt Cell Imaging Shared Resource, Mouse Metabolic Phenotyping Center, Research Electron Microscopy Facility, and the Diabetes Research and Training Center. 
Funding: This work was supported by NIH grants R01DK054902, R37DK050277, and U24DK059637 to DHW; American Heart Association grant 18SFRN33960210 to DHW; and NIH grants F31DK109594 and T32DK007563 to IMW.

\section{References}

1. Rabinowitz D, and Zierler KL (1962) Forearm metabolism in obesity and its response to intraarterial insulin. Characterization of insulin resistance and evidence for adaptive hyperinsulinism. J. Clin. Invest 41, 2173-81 [PubMed: 13972875]

2. Laakso M, Edelman SV, Brechtel G, and Baron AD (1990) Decreased effect of insulin to stimulate skeletal muscle blood flow in obese man. A novel mechanism for insulin resistance. J. Clin. Invest 85, 1844-52 [PubMed: 2189893]

3. DeFronzo RA, and Tripathy D (2009) Skeletal muscle insulin resistance is the primary defect in type 2 diabetes. Diabetes Care. 32 Suppl 2, S157-63 [PubMed: 19875544]

4. Chen YL, and Messina EJ (1996) Dilation of isolated skeletal muscle arterioles by insulin is endothelium dependent and nitric oxide mediated. Am. J. Physiol 270, H2120-4 [PubMed: 8764264]

5. Barrett EJ, Wang H, Upchurch CT, and Liu Z (2011) Insulin regulates its own delivery to skeletal muscle by feed-forward actions on the vasculature. AJP Endocrinol. Metab 301, E252-E263

6. Williams IM, Valenzuela FA, Kahl SD, Ramkrishna D, Mezo AR, Young JD, Wells KS, and Wasserman DH (2018) Insulin exits skeletal muscle capillaries by fluid-phase transport. J. Clin. Invest 128, 699-714 [PubMed: 29309051]

7. Steil GM, Ader M, Moore DM, Rebrin K, and Bergman RN (1996) Transendothelial insulin transport is not saturable in vivo. No evidence for a receptor-mediated process. J. Clin. Invest 97 , 1497-503 [PubMed: 8617883]

8. Rasio E (1982) The Capillary Barrier to Circulating Insulin. Diabetes Care. 5, 158-161 [PubMed: 6756835]

9. Yang YJ, Hope ID, Ader M, and Bergman RN (1989) Insulin transport across capillaries is rate limiting for insulin action in dogs. J. Clin. Invest 84, 1620-8 [PubMed: 2681272]

10. Boucher J, Kleinridders A, and Kahn CR (2014) Insulin receptor signaling in normal and insulinresistant states. Cold Spring Harb. Perspect. Biol 10.1101/cshperspect.a009191

11. King PA, Horton ED, Hirshman MF, and Horton ES (1992) Insulin resistance in obese Zucker rat (fa/fa) skeletal muscle is associated with a failure of glucose transporter translocation. J. Clin. Invest 90, 1568-1575 [PubMed: 1401086]

12. Zierath JR, Houseknecht KL, Gnudi L, and Kahn BB (1997) High-fat feeding impairs insulinstimulated GLUT4 recruitment via an early insulin-signaling defect. Diabetes. 46, 215-23 [PubMed: 9000697]

13. Samuel VT, and Shulman GI (2012) Mechanisms for Insulin Resistance: Common Threads and Missing Links. Cell. 148, 852-871 [PubMed: 22385956]

14. Muoio DMM, and Neufer PDD (2012) Lipid-induced mitochondrial stress and insulin action in muscle. Cell Metab 15, 595-605 [PubMed: 22560212]

15. Wu H, and Ballantyne CM (2017) Skeletal muscle inflammation and insulin resistance in obesity. J. Clin. Invest 127, 43-54 [PubMed: 28045398]

16. Gudbjörnsdóttir S, Sjöstrand M, Strindberg L, Wahren J, and Lönnroth P (2003) Direct measurements of the permeability surface area for insulin and glucose in human skeletal muscle. J. Clin. Endocrinol. Metab 88, 4559-64 [PubMed: 14557422]

17. Bergman RN (2003) Insulin action and distribution of tissue blood flow. J. Clin. Endocrinol. Metab 88, 4556-8 [PubMed: 14557421]

18. Sjöstrand M, Gudbjörnsdottir S, Holmäng A, Lönn L, Strindberg L, and Lönnroth P (2002) Delayed transcapillary transport of insulin to muscle interstitial fluid in obese subjects. Diabetes. 51, 2742-8 [PubMed: 12196467]

19. Kubota T, Kubota N, Kumagai H, Yamaguchi S, Kozono H, Takahashi T, Inoue M, Itoh S, Takamoto I, Sasako T, Kumagai K, Kawai T, Hashimoto S, Kobayashi T, Sato M, Tokuyama K, Nishimura S, Tsunoda M, Ide T, Murakami K, Yamazaki T, Ezaki O, Kawamura K, Masuda H, 
Moroi M, Sugi K, Oike Y, Shimokawa H, Yanagihara N, Tsutsui M, Terauchi Y, Tobe K, Nagai R, Kamata K, Inoue K, Kodama T, Ueki K, and Kadowaki T (2011) Impaired insulin signaling in endothelial cells reduces insulin-induced glucose uptake by skeletal muscle. Cell Metab 13, 294307 [PubMed: 21356519]

20. Broussard JL, Castro AVB, Iyer M, Paszkiewicz RL, Bediako IA, Szczepaniak LS, Szczepaniak EW, Bergman RN, and Kolka CM (2016) Insulin access to skeletal muscle is impaired during the early stages of diet-induced obesity. Obesity. 24, 1922-1928 [PubMed: 27569119]

21. Castillo C, Bogardus C, Bergman R, Thuillez P, and Lillioja S (1994) Interstitial insulin concentrations determine glucose uptake rates but not insulin resistance in lean and obese men. J. Clin. Invest 93, 10-6 [PubMed: 8282776]

22. Gudbjörnsdóttir S, Sjöstrand M, Strindberg L, and Lönnroth P (2005) Decreased muscle capillary permeability surface area in type 2 diabetic subjects. J. Clin. Endocrinol. Metab 90, 1078-82 [PubMed: 15536160]

23. Winzell MS, Ahrén B, and Ahren B (2004) The High-Fat Diet-Fed Mouse: A Model for Studying Mechanisms and Treatment of Impaired Glucose Tolerance and Type 2 Diabetes. Diabetes. 53 Suppl 3, S215-9 [PubMed: 15561913]

24. Williams IM, Otero YF, Bracy DP, Wasserman DH, Biaggioni I, and Arnold AC (2016) Chronic Angiotensin-(1-7) Improves Insulin Sensitivity in High-Fat Fed Mice Independent of Blood Pressure. Hypertension. 67, 983-91 [PubMed: 26975707]

25. Tabula Muris Consortium, Overall coordination, Logistical coordination, Organ collection and processing, Library preparation and sequencing, Computational data analysis, Cell type annotation, Writing group, Supplemental text writing group, and Principal investigators (2018) Single-cell transcriptomics of 20 mouse organs creates a Tabula Muris. Nature. 10.1038/ s41586-018-0590-4

26. Muniyappa R, and Sowers JR (2013) Role of insulin resistance in endothelial dysfunction. Rev. Endocr. Metab. Disord 14, 5-12 [PubMed: 23306778]

27. Williams IM, McClatchey PM, Bracy DP, Valenzuela FA, and Wasserman DH (2018) Acute Nitric Oxide Synthase Inhibition Accelerates Trans-Endothelial Insulin Efflux in Vivo. Diabetes. $10.2337 / \mathrm{db} 18-0288$

28. Lee J-F, Gordon S, Estrada R, Wang L, Siow DL, Wattenberg BW, Lominadze D, and Lee M-J (2009) Balance of S1P1 and S1P2 signaling regulates peripheral microvascular permeability in rat cremaster muscle vasculature. Am. J. Physiol. Heart Circ. Physiol 296, H33-42 [PubMed: 19011048]

29. Albelda SM, Sampson PM, Haselton FR, McNiff JM, Mueller SN, Williams SK, Fishman AP, and Levine EM (1988) Permeability characteristics of cultured endothelial cell monolayers. J. Appl. Physiol 64, 308-22 [PubMed: 2451657]

30. Lacorre D-A, Baekkevold ES, Garrido I, Brandtzaeg P, Haraldsen G, Amalric F, and Girard J-P (2004) Plasticity of endothelial cells: rapid dedifferentiation of freshly isolated high endothelial venule endothelial cells outside the lymphoid tissue microenvironment. Blood. 103, 4164-4172 [PubMed: 14976058]

31. Durr E, Yu J, Krasinska KM, Carver LA, Yates JR, Testa JE, Oh P, and Schnitzer JE (2004) Direct proteomic mapping of the lung microvascular endothelial cell surface in vivo and in cell culture. Nat. Biotechnol 22, 985-92 [PubMed: 15258593]

32. Yen TT, Stienmetz J, and Simpson PJ (1970) Blood volume of obese (ob-ob) and diabetic (db-db) mice. Proc. Soc. Exp. Biol. Med 133, 307-8 [PubMed: 5412353]

33. Predescu SA, Predescu DN, and Malik AB (2007) Molecular determinants of endothelial transcytosis and their role in endothelial permeability. Am. J. Physiol. Cell. Mol. Physiol 293, L823-42

34. Cassuto J, Dou H, Czikora I, Szabo A, Patel VS, Kamath VV, Belin de Chantemele E, Feher A, Romero MJ, and Bagi Z (2013) Peroxynitrite Disrupts Endothelial Caveolae Leading to eNOS Uncoupling and Diminished Flow-Mediated Dilation in Coronary Arterioles of Diabetic Patients. Diabetes. 63, 1381-1393 [PubMed: 24353182] 
35. Wang H, Liu Z, Li G, and Barrett EJ (2006) The vascular endothelial cell mediates insulin transport into skeletal muscle. Am. J. Physiol. Endocrinol. Metab 291, E323-32 [PubMed: 16569759]

36. Wang H, Wang AX, and Barrett EJ (2011) Caveolin-1 is required for vascular endothelial insulin uptake. Am. J. Physiol. Endocrinol. Metab 300, E134-44 [PubMed: 20959538]

37. Wang H, Wang AX, Aylor K, and Barrett EJ (2015) Caveolin-1 phosphorylation regulates vascular endothelial insulin uptake and is impaired by insulin resistance in rats. Diabetologia. 58, 1344-53 [PubMed: 25748795]

38. Kang L, Ayala JE, Lee-Young RS, Zhang Z, James FD, Neufer PD, Pozzi A, Zutter MM, and Wasserman DH (2011) Diet-induced muscle insulin resistance is associated with extracellular matrix remodeling and interaction with integrin alpha2beta1 in mice. Diabetes. 60, 416-26 [PubMed: 21270253]

39. Kang L, Lantier L, Kennedy A, Bonner JS, Mayes WH, Bracy DP, Bookbinder LH, Hasty AH, Thompson CB, and Wasserman DH (2013) Hyaluronan accumulates with high-fat feeding and contributes to insulin resistance. Diabetes. 62, 1888-96 [PubMed: 23349492]

40. Bendayan M, and Rasio EA (1996) Transport of insulin and albumin by the microvascular endothelium of the rete mirabile. J. Cell Sci 109 ( Pt 7, 1857-64 [PubMed: 8832408]

41. Rippe B, Rosengren B-I, Carlsson O, and Venturoli D (2002) Transendothelial Transport: The Vesicle Controversy. J. Vasc. Res 39, 375-390 [PubMed: 12297701]

42. Natarajan KN, Miao Z, Jiang M, Huang X, Zhou H, Xie J, Wang C, Qin S, Zhao Z, Wu L, Yang N, Li B, Hou Y, Liu S, and Teichmann SA (2019) Comparative analysis of sequencing technologies for single-cell transcriptomics. Genome Biol 20, 70 [PubMed: 30961669]

43. Jaldin-Fincati JR, Pereira RVS, Bilan PJ, and Klip A (2018) Insulin uptake and action in microvascular endothelial cells of lymphatic and blood origin. Am. J. Physiol. Metab 10.1152/ ajpendo.00008.2018

44. Pries AR, Ley K, Claassen M, and Gaehtgens P (1989) Red cell distribution at microvascular bifurcations. Microvasc. Res 38, 81-101 [PubMed: 2761434]

45. Yang Y, Smith DL, Keating KD, Allison DB, Nagy TR, and Nagy TR (2014) Variations in body weight, food intake and body composition after long-term high-fat diet feeding in C57BL/6J mice. Obesity (Silver Spring). 22, 2147-55 [PubMed: 24942674] 


\section{Study Importance}

\section{What is already known about this subject?}

- Humans with obesity and insulin resistance show reduced delivery of insulin to the skeletal muscle interstitial space.

- Insulin delivery is controlled by skeletal muscle perfusion and transendothelial insulin transport.

- Endothelial function and skeletal muscle perfusion are impaired in the setting of obesity, insulin resistance and type 2 diabetes.

\section{What does this study add?}

- Trans-endothelial insulin transport is delayed in skeletal muscle capillaries of male mice made obese and insulin resistant by high fat feeding.

- $\quad$ Skeletal muscle capillary endothelia of DIO male mice have a reduction in endothelial vesicles, the putative vehicles for insulin transport.

- $\quad$ Female mice are protected from the effects of HFD on adiposity, insulin resistance, trans-endothelial insulin transport, and endothelial ultrastructure. 


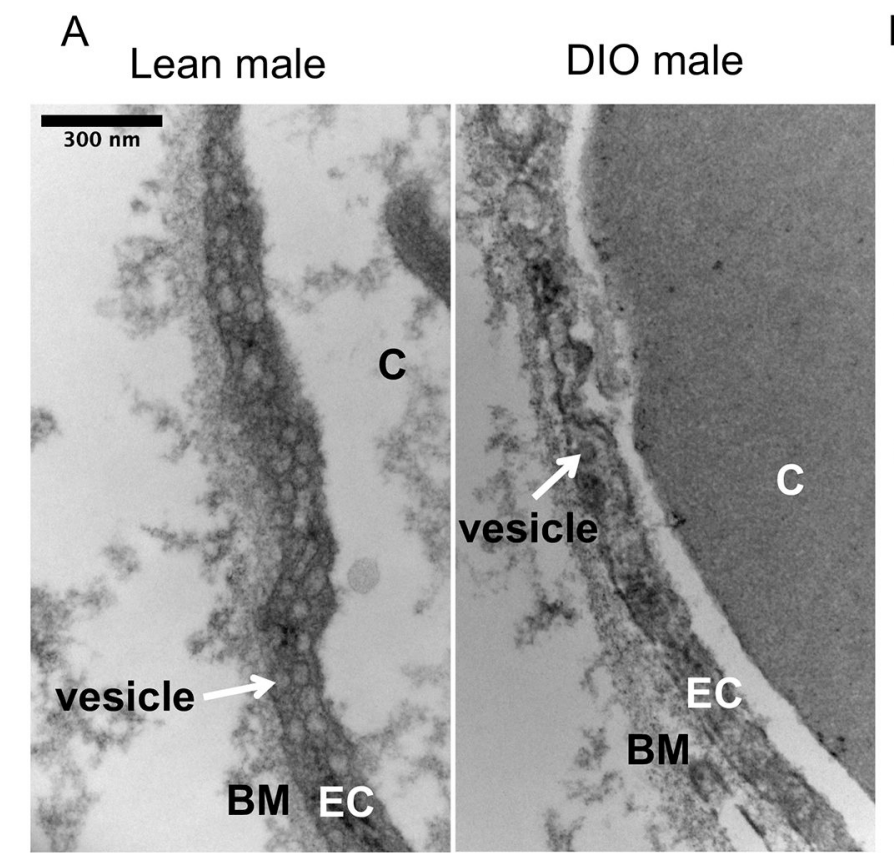

B
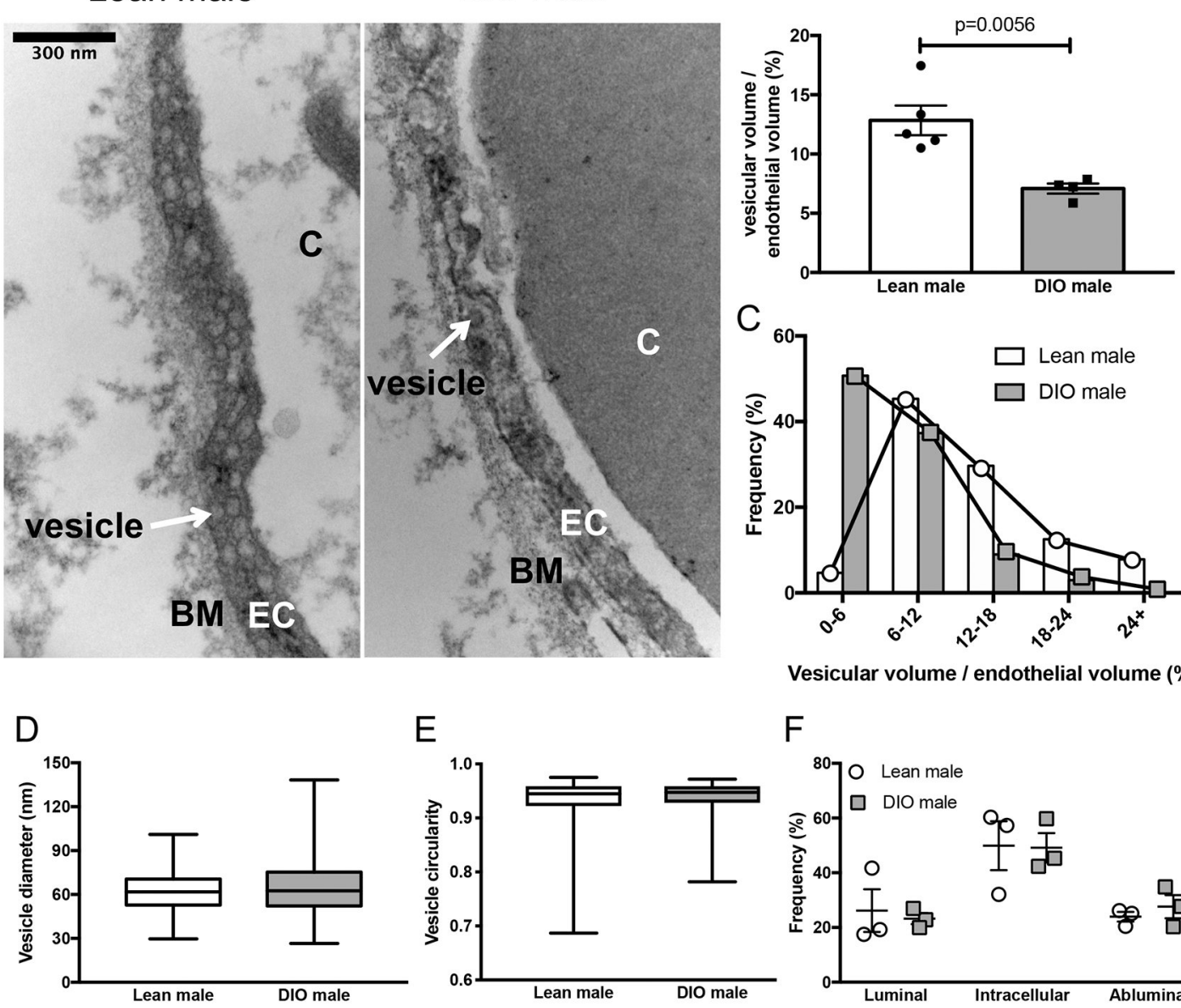

$\mathrm{E}$

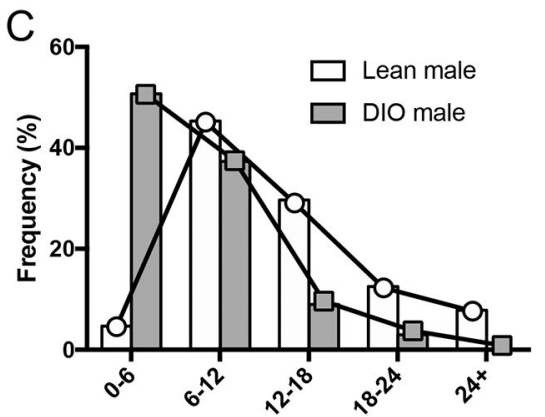

Vesicular volume / endothelial volume (\%)

G

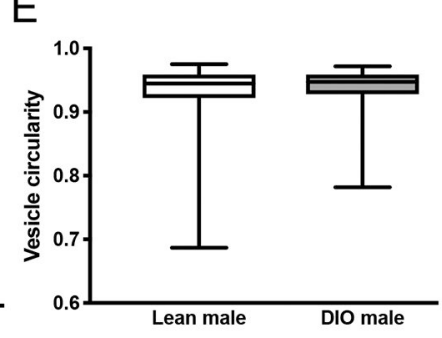

$\mathrm{F}$
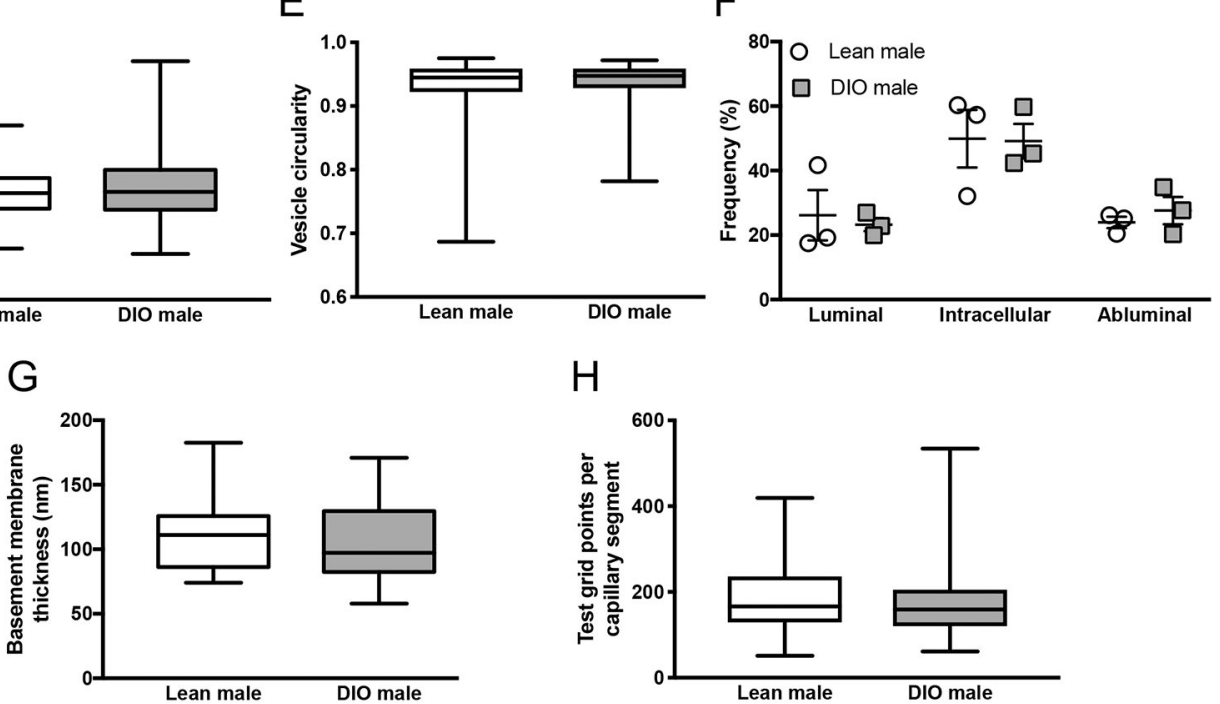

$\mathrm{H}$

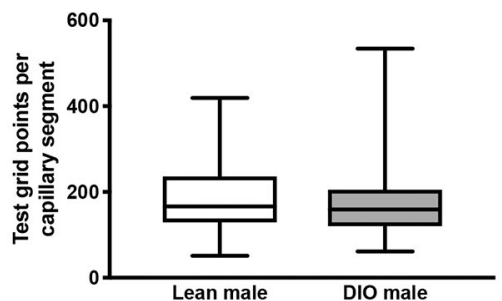

Figure 1: Skeletal muscle capillaries of DIO male mice contain fewer endothelial vesicles than lean mice.

A) Representative electron micrographs of the capillary endothelium in the gastrocnemius of lean and DIO male mice. B) Volume of vesicles relative to total endothelial volume in lean $(n=5)$ and DIO $(n=4)$ male mice. C) Frequency distribution of endothelial vesicle densities in all capillaries grouped from lean $(n=64)$ and DIO male mice $(n=66)$. D) The average diameter of all endothelial vesicles in lean $(n=227)$ and DIO $(n=116)$ male mice. E) The circularity of vesicles in lean $(n=146)$ and DIO $(n=88)$ male mice. Circularity values of 1 and 0 indicate perfect circles and very elongated shapes, respectively. F) Frequency distribution of the localization of vesicles in the capillary endothelium. G) Average basement membrane thickness in capillaries from lean $(n=31)$ and DIO male mice $(n=31)$. In 
the box and whisker blots, the box extends from the $25^{\text {th }}$ to the $75^{\text {th }}$ percentiles and the whiskers indicate the range. $\mathbf{H})$ The number of test grid points counted within each capillary segment in lean $(n=184)$ and DIO $(n=181)$ male mice, an index of endothelial volume. Groups were compared using Student's t-test. C - capillary lumen, EC - endothelial cell, $\mathrm{BM}$ - basement membrane, DIO - diet-induced obese. 
A

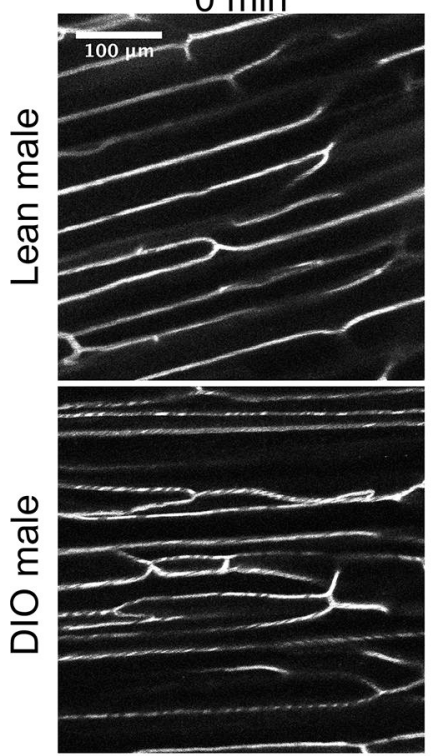

B
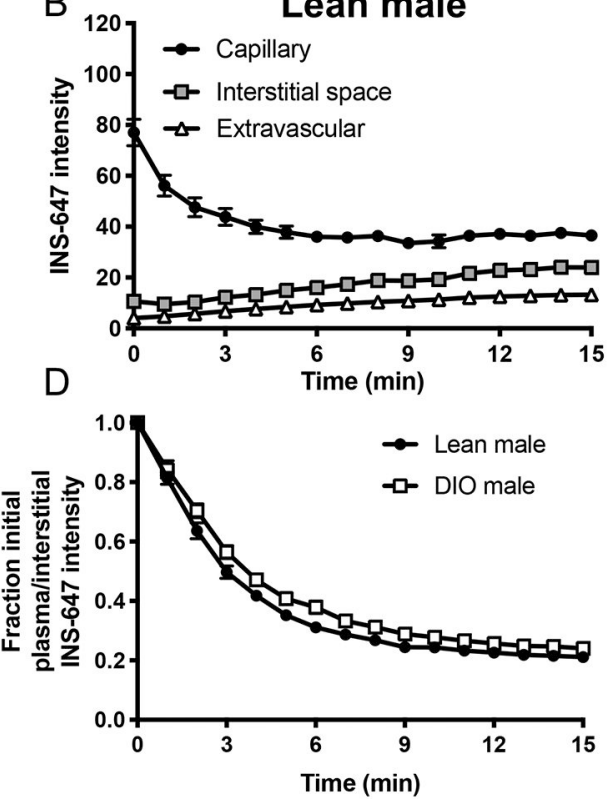

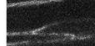

$5 \min$
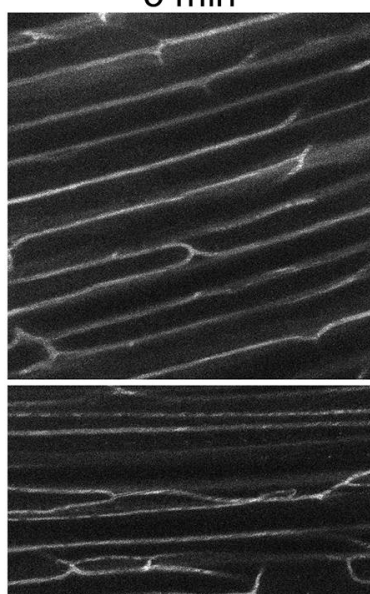
$-$ $>$ $+2$

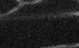
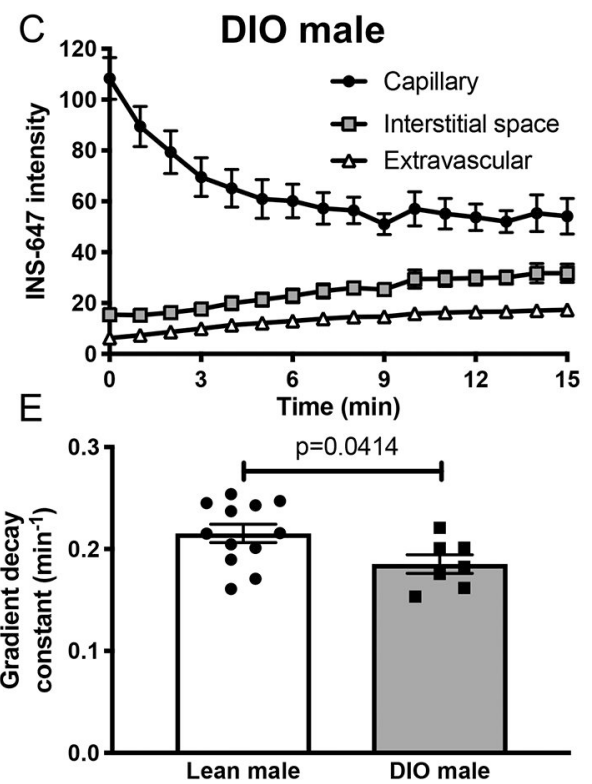

Figure 2: Obese male mice have impaired trans-endothelial insulin transport in skeletal muscle capillaries.

A) Representative INS-647 images (maximum intensity projections) in lean ( $\mathrm{n}=12)$ and DIO $(n=7)$ male mice. B-C) Capillary, interstitial, and total extravascular INS-647 in the field of view as a function of time following the beginning of imaging in $\mathbf{B}$ ) lean and C) DIO male mice. $\mathrm{T}=0$ min indicates the beginning of imaging which occurs $\sim 15$ seconds after injection of INS-647. The interstitial space is defined as the region emanating $1-3 \mu \mathrm{m}$ from the capillary wall. D) The ratio of plasma to interstitial INS-647 as a function of time following INS-647 injection, normalized to the ratio at $\mathrm{t}=0 \mathrm{~min}$. E) Decay constant of the plasma / interstitial INS-647 ratio, a measure of trans-endothelial insulin transport kinetics. Groups were compared using Student's t-test. INS-647 - insulin-647. 
A
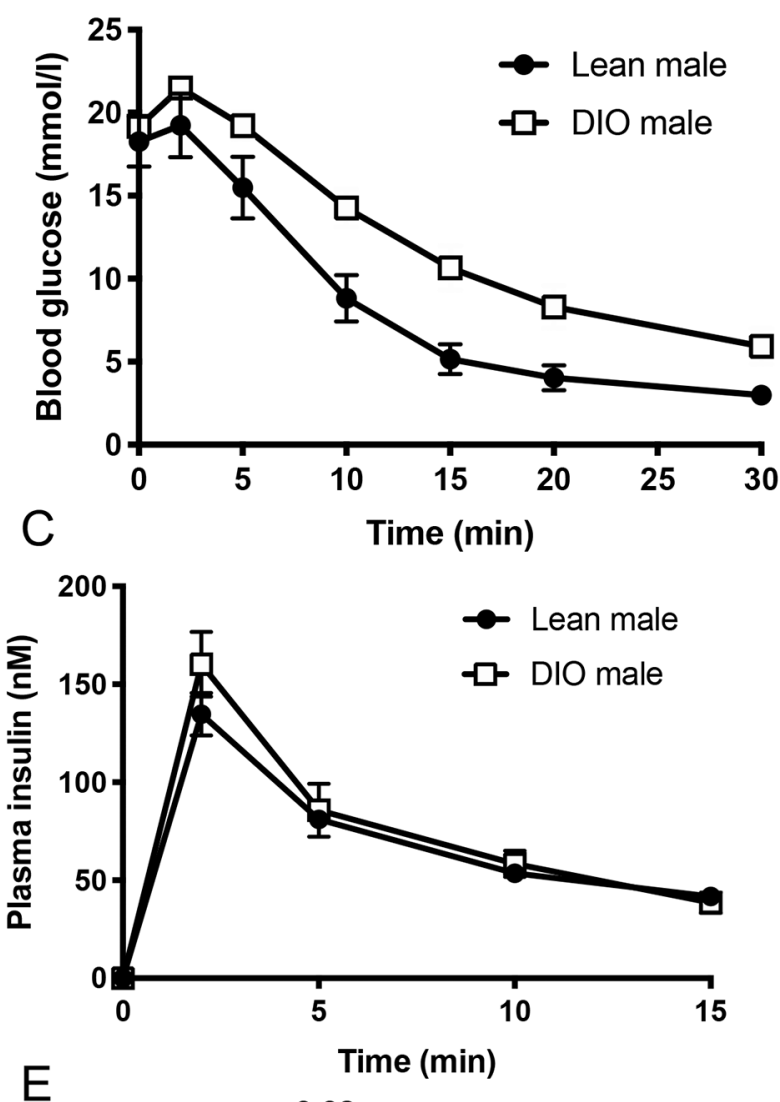

E

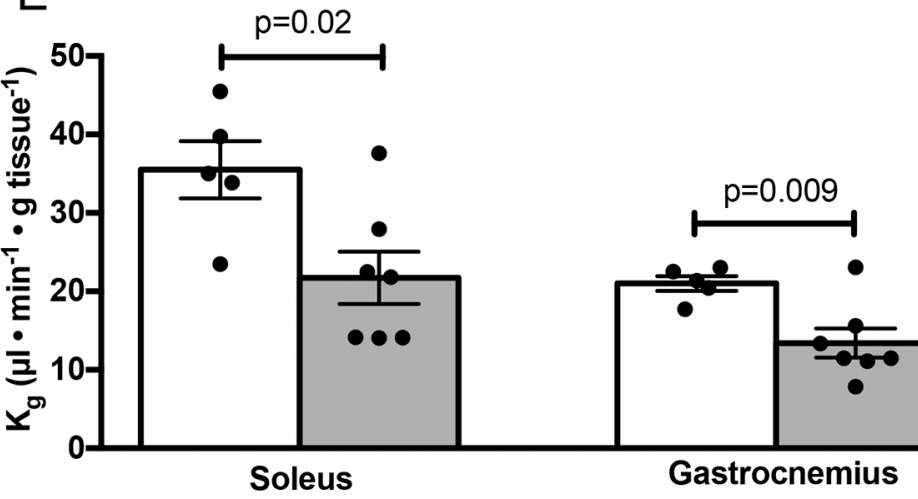

B

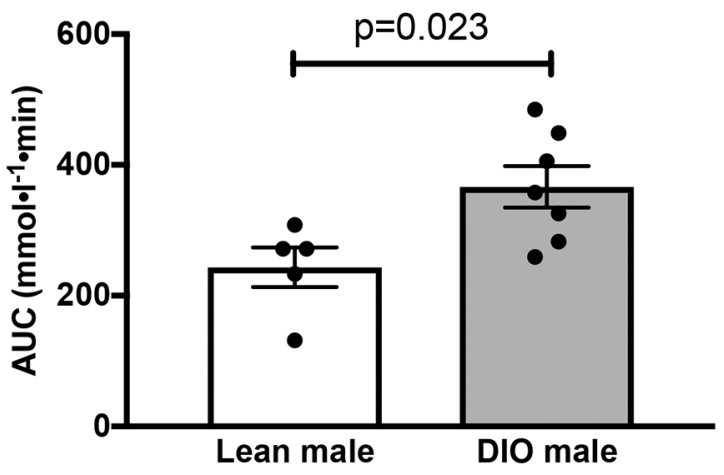

D

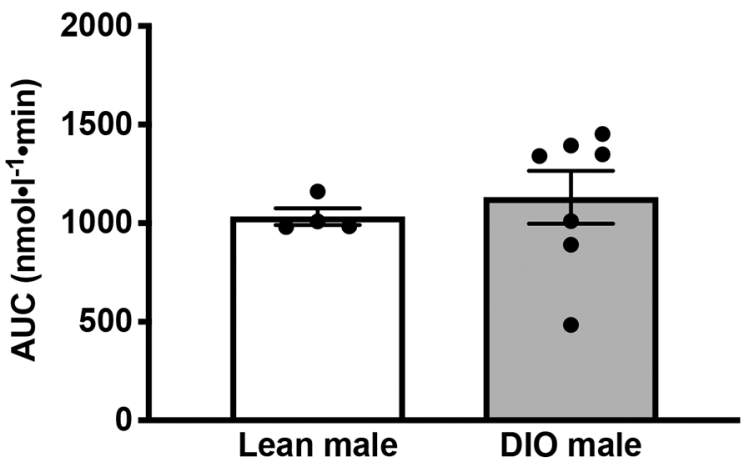

Figure 3: DIO male mice display skeletal muscle insulin resistance.

A) Glucose excursions in anesthetized lean $(n=5)$ and DIO $(n=7)$ male mice following a $4 \mathrm{U} / \mathrm{kg}$ intravenous insulin bolus. B) Area under the glucose excursion curves in A. C) Plasma insulin excursions in lean $(n=4)$ and DIO $(n=7)$ male mice following a $4 \mathrm{U} / \mathrm{kg}$ intravenous insulin bolus. D) Area under the insulin excursion curves in C. E) Clearance of $2\left[{ }^{14} \mathrm{C}\right]$ deoxyglucose during the insulin tolerance tests by the soleus, gastrocnemius, and vastus muscles. Groups were compared using Student's t-test. 

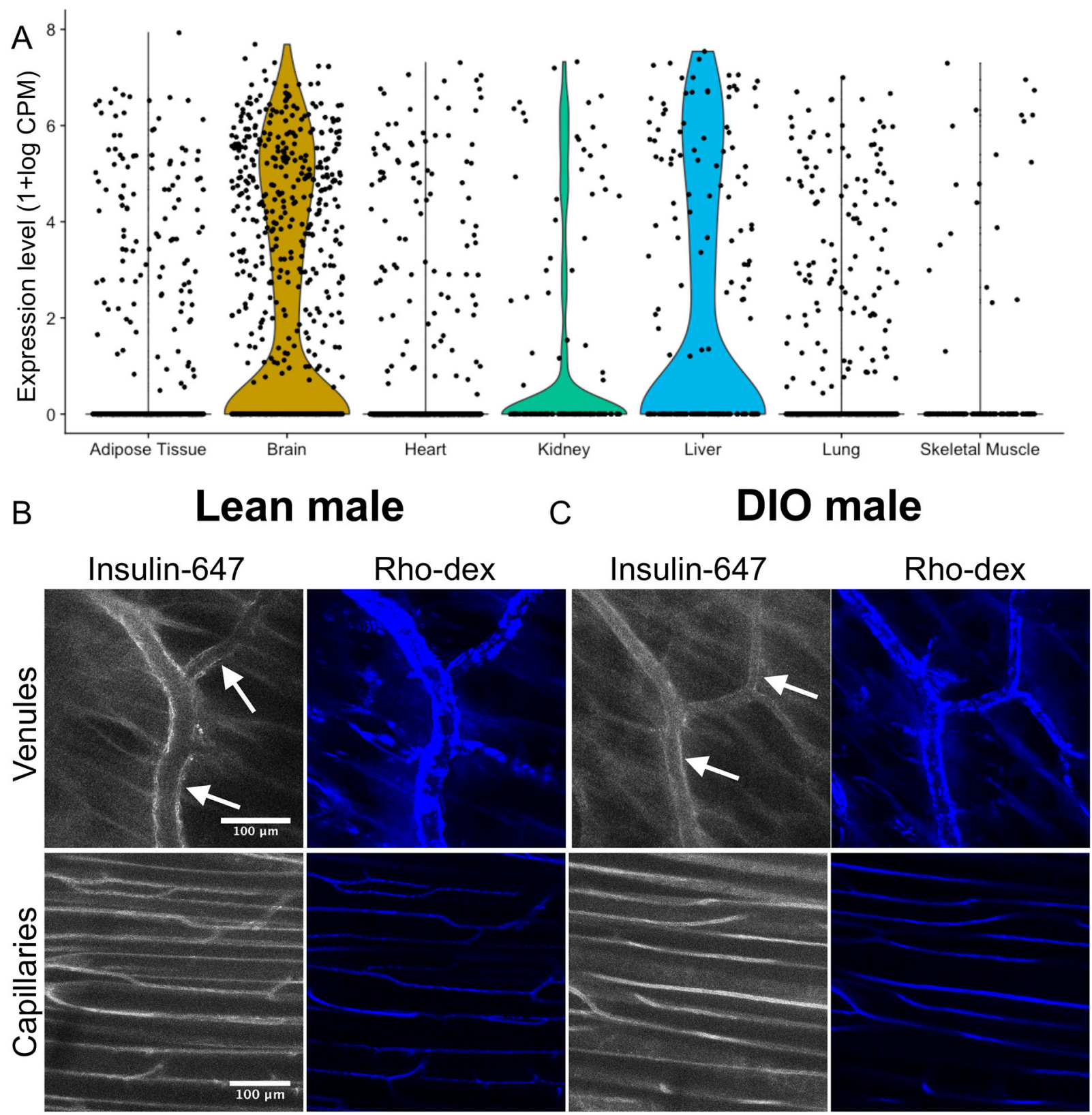

Figure 4: Capillary trans-endothelial insulin transport in skeletal muscle does not involve the insulin receptor or endothelial insulin accumulation.

A) Violin plots of insulin receptor mRNA expression in tissue-specific endothelial cells as determined by single-cell RNA sequencing. B,C) Intravital microscopy images of INS-647 and rhodamine-labeled 2MDa dextran (Rho-dex) in venules (top panels) and capillaries (bottom panels) from B) lean and C) DIO male mice. Both INS-647 and rho-dex can be seen accumulating in the endothelium of venules but not capillaries. Arrows indicate regions of insulin accumulation in the venular endothelium. Rho-dex - 2MDa tetramethylrhodaminedextran, CPM - counts per million. 

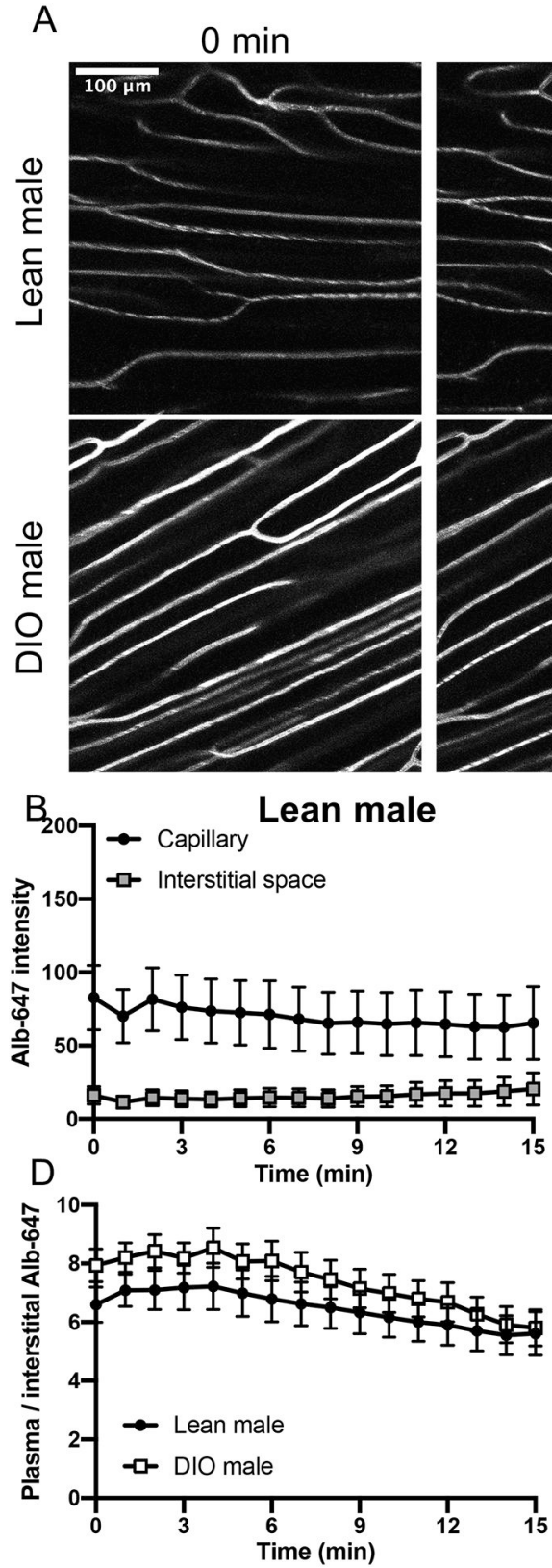

$7 \min$
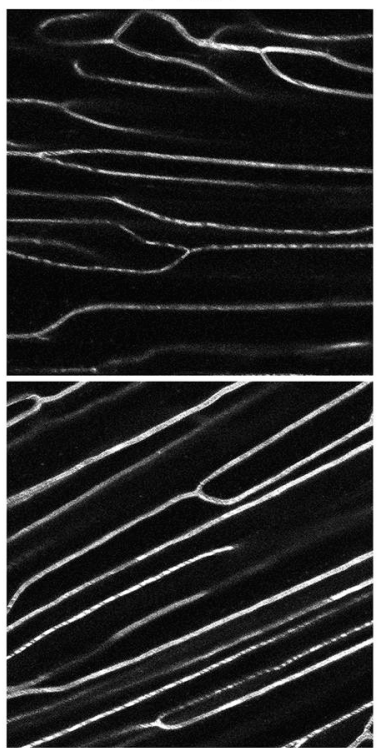

C
$15 \min$

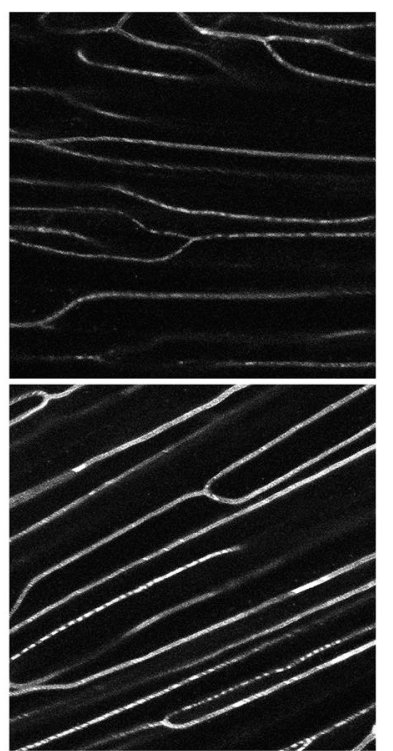

DIO male
$\mathrm{E}$
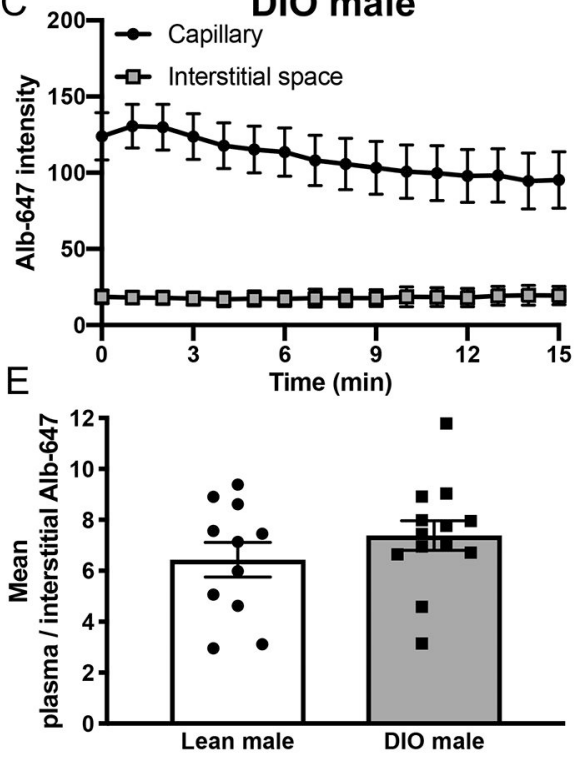

Figure 5: No difference in albumin equilibration between lean and DIO male mice.

A) Representative Alb-647 images (maximum intensity projections) in lean ( $\mathrm{n}=11)$ and DIO $(n=13)$ male mice. B-C) Capillary and interstitial Alb-647 intensity as a function of time following injection in B) lean and C) DIO male mice. The interstitial space is defined as the

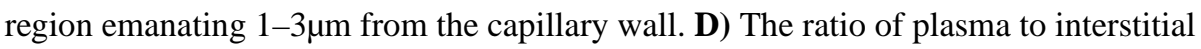
Alb-647 as a function of time following Alb-647 injection. E) Mean plasma to interstitial Alb-647 ratio over the course of the experiment. Groups were compared using Student's ttest. Alb-647 - albumin-647. 


\section{A \\ HFD female}

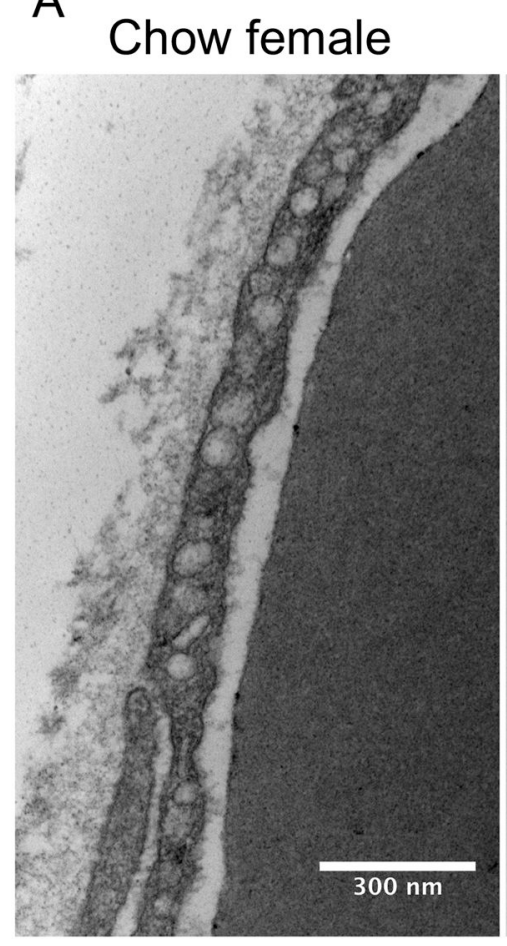

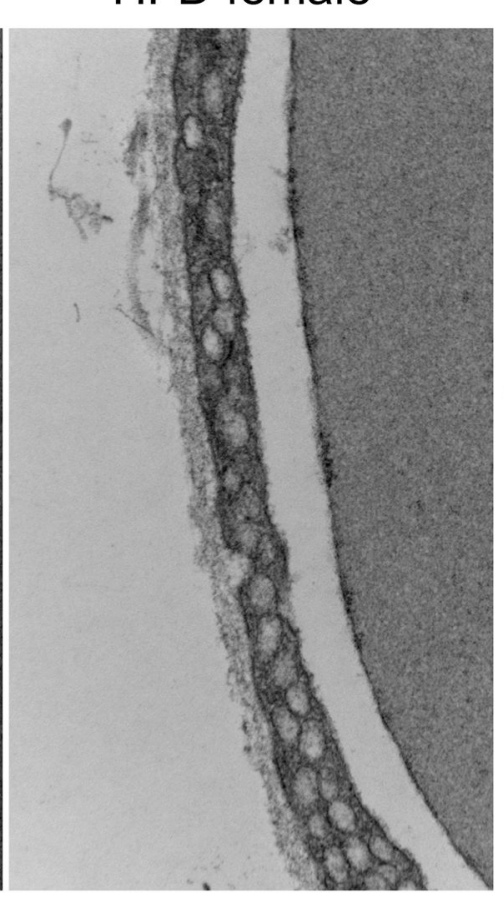

B

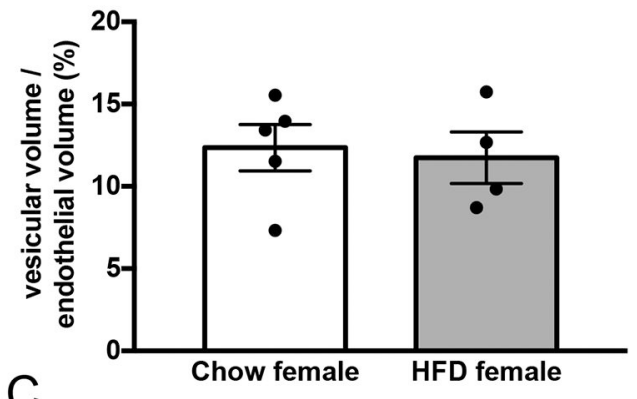

C

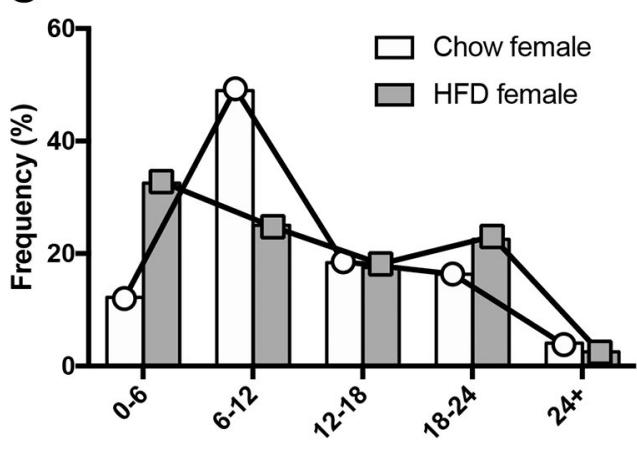

Vesicular volume / endothelial volume (\%)

Figure 6: No effect of HFD on endothelial vesicles in female mice.

A) Representative electron micrographs of the capillary endothelium in the gastrocnemius of chow and HFD-fed female mice. B) Volume of vesicles relative to total endothelial volume in chow $(n=5)$ and HFD-fed $(n=4)$ female mice. C) Frequency distribution of endothelial vesicular densities in all capillaries pooled from chow $(n=49)$ and HFD-fed female mice $(n=40)$. Groups were compared by Student's t-test. 

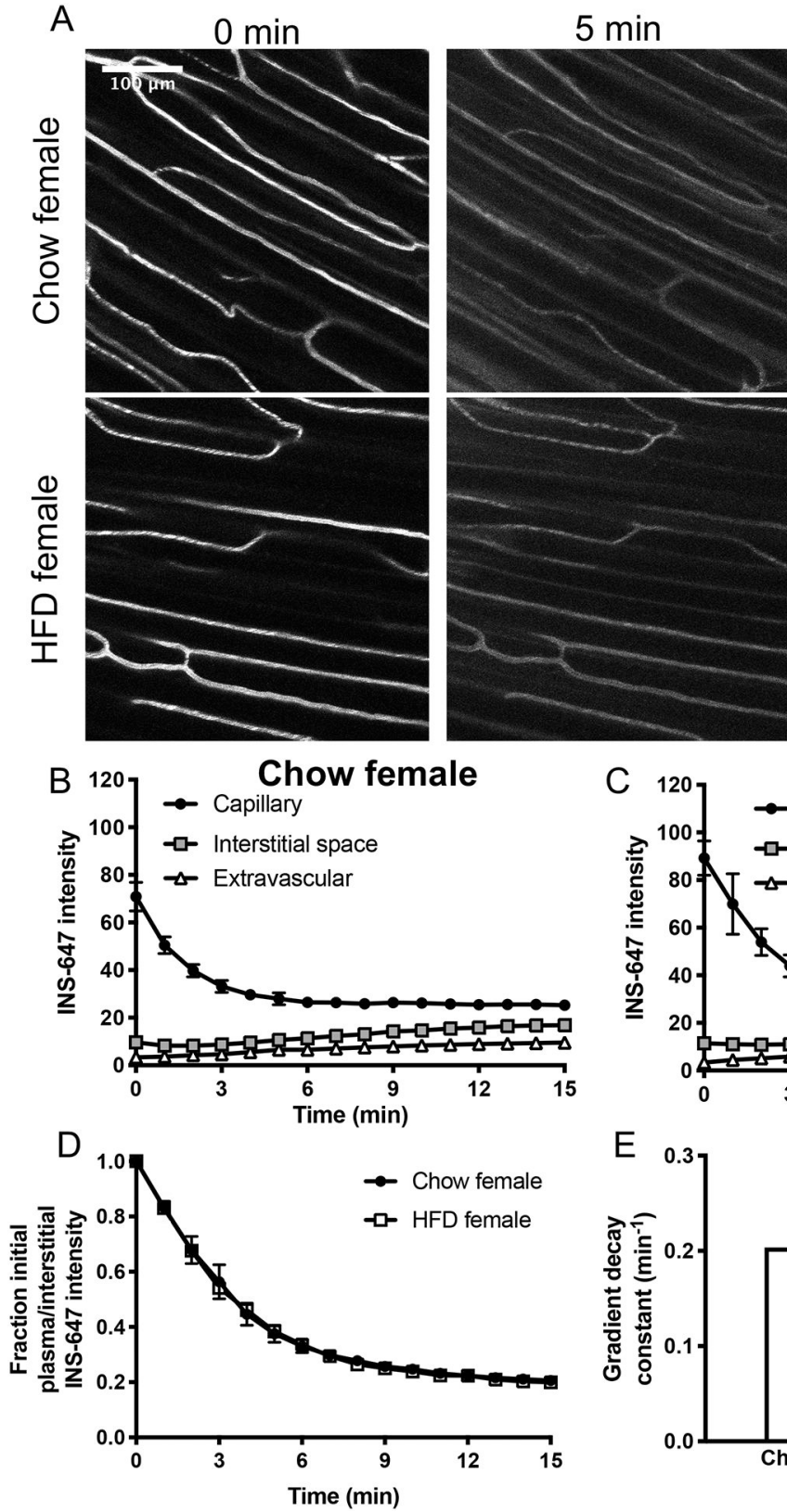
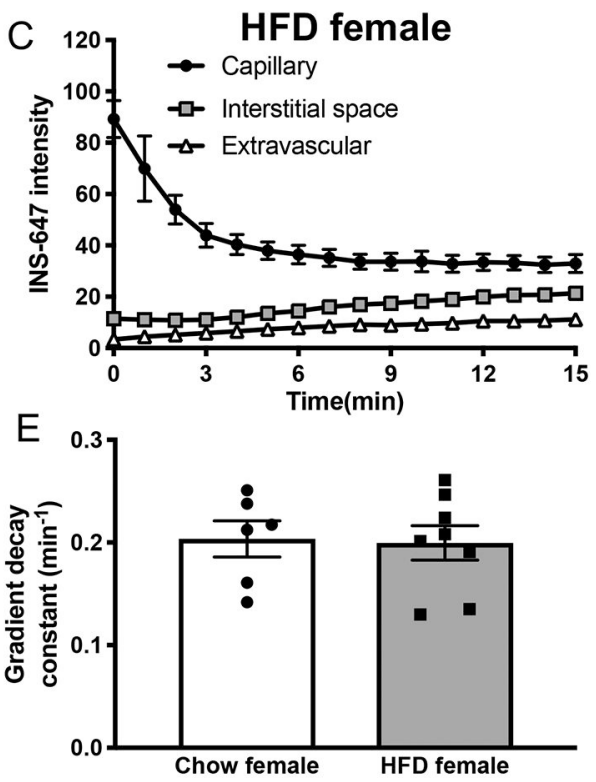

Figure 7: HFD does not alter trans-endothelial insulin transport in females.

A) Representative INS-647 images (maximum intensity projections) in chow (n=6) and HFD-fed ( $\mathrm{n}=8$ ) female mice. B-C) Capillary, interstitial, and total extravascular INS-647 in the field of view as a function of time following injection in $\mathbf{B}$ ) chow and $\mathbf{C}$ ) HFD-fed female mice. The interstitial space is defined as the region emanating $1-3 \mu \mathrm{m}$ from the capillary wall. D) The ratio of plasma to interstitial INS-647 as a function of time following INS-647 injection, normalized to the ratio at $\mathrm{t}=0 \mathrm{~min}$. E) The gradient decay constant of the plasma to interstitial INS-647 ratio as a function of time. Groups were compared using Student's t-test. 
Table 1.

Effects of HFD on insulin and albumin kinetics in male and female mice.

\begin{tabular}{|l|l|l|l|l|}
\hline Parameter & Comparison & $\mathbf{P}_{\text {Time }}$ & $\mathbf{P}_{\text {Diet }}$ & $\mathbf{P}_{\text {Interaction }}$ \\
\hline Capillary INS-647 & Lean vs. DIO male & $<0.0001$ & 0.0006 & $<0.0001$ \\
\hline Interstitial INS-647 & Lean vs. DIO male & $<0.0001$ & 0.0103 & 0.3779 \\
\hline Extravascular INS-647 & Lean vs. DIO male & $<0.0001$ & 0.0089 & 0.0852 \\
\hline Capillary Alb-647 & Lean vs. DIO male & $<0.0001$ & 0.1162 & 0.2070 \\
\hline Interstitial Alb-647 & Lean vs. DIO male & 0.2210 & 0.7796 & 0.9928 \\
\hline Capillary INS-647 & Chow vs. HFD female & $<0.0001$ & 0.0569 & 0.4977 \\
\hline Interstitial INS-647 & Chow vs. HFD female & $<0.0001$ & 0.1852 & 0.8955 \\
\hline Extravascular INS-647 & Chow vs. HFD female & $<0.0001$ & 0.3370 & 0.7199 \\
\hline
\end{tabular}

Effects of HFD on the levels and kinetics INS-647 in the plasma, interstitium, and extravascular space in male and female mice. Table also shows the effects of HFD on the levels and kinetics of Alb-647 in the plasma and interstitial space in male mice. 2-way repeated measures ANOVA was used to calculate p-values for the effect of time (PTime), diet ( $\mathrm{P}_{\text {diet }}$ ), and their interaction ( $\mathrm{P}_{\text {interaction }}$ ) for each of these parameters. HFD high-fat diet, INS-647 - insulin-647, DIO - diet-induced obese, Alb-647 - albumin-647. 